\title{
Educacion formal y deporte de élite en adolescentes argentinos
}

Education and elite sports in Argentinean adolescents

\author{
Fernando Alberto Laiño \\ Departamento de Humanidades y Ciencias Sociales. \\ Universidad Nacional de La Matanza, Argentina
}

\author{
Ianina Tuñón \\ Departamento de Humanidades y Ciencias Sociales. \\ Universidad Nacional de La Matanza, Argentina \\ tunon.ianina@gmail.com
}

\author{
Hernán Castro \\ Departamento de Humanidades y Ciencias Sociales. \\ Universidad Nacional de La Matanza, Argentina \\ Martin Pérez Rodriguez \\ Departamento de Humanidades y Ciencias Sociales. \\ Universidad Nacional de La Matanza, Argentina
}

\section{Resumen:}

La adolescencia es el período más favorable para detectar, entrenar y desarrollar el potencial de los alumnos/as con talento deportivo. El objetivo de este trabajo es identificar barreras y facilitadores escolares para los representantes de Argentina en los Juegos Olímpicos de la Juventud 2018. Participaron del estudio 408 sujetos de 14 a 17 años de edad (249 varones y 159 mujeres) preseleccionados. Se aplicaron algunos indicadores de la Encuesta para Atletas de Elite (Irlanda del Norte), relacionados con el deporte practicado y la escuela. Se calcularon las frecuencias porcentuales para todas las variables y se realizaron contrastes en función de sexo, lugar de residencia y tipo de deporte practicado mediante pruebas U de Mann-Whitney y Kruskal-Wallis ( $\mathrm{p}$ $<0.05)$. Se destaca el rol asumido por los clubes como espacio de inicio y de práctica actual del deporte. En cuanto a distintos reconocimientos de la escuela hacia los talentos, es mayor en residentes de Ciudad de Buenos Aires que en otras jurisdicciones. Se identifica la necesidad de mayor soporte a los deportes individuales, y fortalecer el apoyo de la comunidad educativa a los talentos deportivos, y el diseño y ejecución de políticas públicas de sostén y desarrollo de estos.

Palabras clave: Talentos Deportivos, Escuela Secundaria, Facilitadores, Educación Deportiva, Políticas Públicas.

\begin{abstract}
:
Adolescence is the most favorable period to detect, train and develop the potential of students with sports talent. The aim of this paper is to identify barriers and school facilitators for Argentina's representatives in 2018 Youth Olympic Games. 408 preselected participants from 14 to 17 years old ( 249 males and 159 females) participated in the study. Some indicators of the Elite Athletes Survey (Northern Ireland) related to practiced sport and school were applied. The percentage frequencies for all the variables were calculated and contrasts were made based on sex, place of residence and type of sport practiced by U-Mann-Whitney and KruskalWallis tests $(p<0.05)$. The role assumed by clubs as a starting space and current practice of sport is highlighted. As for different recognitions of the school towards talents, it is greater in residents of the City of Buenos Aires than in other jurisdictions. We identify the need for greater support for individual sports, and the need to strengthen the support of educational community for sports talents, and to design and implement public policies to assist and develop them.
\end{abstract}

KEYwORDs: Sports Talents, Middle and High School, Facilitators, Sports Education, Public Policies. 


\section{INTRODUCCIÓN}

En el marco de los Juegos Olímpicos de la Juventud 2018 en Buenos Aires, se realizó una amplia convocatoria nacional buscando talentos deportivos (Farinola, Tuñón, Laíño, Marchesich \& Pérez Rodríguez, 2018). En la etapa final del proceso de entrenamiento y selección del grupo que representaría al país, se realizó una encuesta procurando identificar múltiples aspectos sociales y culturales que caracterizaban a esta población. En el marco de esta encuesta, se indagó sobre aspectos específicos de la relación entre el proceso de especialización deportiva y la escuela.

Más específicamente, se incluyeron indicadores sobre el espacio donde se comenzó a desarrollar el deporte, lugar donde practica el deporte, práctica de deporte durante la clase de Educación Física y participación en competencias deportivas escolares durante la escuela secundaria, que son algunos de los principales aspectos que constituyen un modelo de Educación Deportiva (Siedentop, 1994), con fuerte acento en el nivel secundario de enseñanza.

Según investigaciones que tuvieron por objeto el análisis de las variables que tienen influencia sobre un proceso de enseñanza-aprendizaje de calidad respecto al dictado de los contenidos deportivos en escuelas primarias y secundarias, se concluyó que el deporte en las clases de Educación Física, raramente reproduce las características que lo hacen atractivo, generando cierta irrelevancia en los alumnos; no produciendo un verdadero interés, ni por el deporte, ni por su práctica, fuera del contexto escolar. Además, el aprendizaje y la práctica de las habilidades deportivas se dan de una forma aislada o descontextualizada, planteando dificultades en la transferencia a situaciones de juego real (Siedentop, 1994).

Es preciso considerar que la educación y el deporte tienen demandas conflictivas y, por lo tanto, están en una relación competitiva. En este sentido, Henriksen (2010) destacó que es esencial que la relación del deporte con otras instituciones debe estar coordinada, para que el atleta tenga la oportunidad de desarrollarse en un ambiente estable y seguro (Aalberg \& Sæther, 2016).

Henriksen (2010) señala la importancia de la capacidad de una comunidad exitosa para tener en cuenta el desarrollo general del deportista. Los factores fuera del campo deportivo, son centrales y afectan en gran medida el rendimiento de un atleta. Tener una carrera integral y coherente depende del desarrollo en varios dominios que son interdependientes, y el contexto deportivo no existe separado de la vida real. Se dice que los cambios en las esferas psicológica, psicosocial, académica y ocupacional tienen un impacto decisivo en la carrera de un deportista, y confirman que un atleta que experimenta transiciones centrales en su carrera y en su vida, debe coordinar los desafíos que acompañan para obtener el éxito en el contexto deportivo.

Otros estudios han demostrado que los factores contextuales pueden afectar el resultado del proceso de desarrollo del talento. En un estudio sobre el talento a través de los deportes, se comprobó que los atletas exitosos atribuían en gran medida su éxito a factores contextuales, como un buen ambiente en su club, amigos dentro del deporte, baja presión sobre los logros a una edad temprana y buenas relaciones con sus entrenadores (Aalberg \& Sæther, 2016).

Esto se corresponde bien con el entendimiento del talento como mucho más que un conjunto de características físicas. El potencial de un deportista, en igual medida, consiste en la capacidad del individuo para desarrollar y utilizar sus recursos para hacer frente a los desafíos dentro y fuera del campo deportivo (Aalberg \& Sæther, 2016).

Una investigación cualitativa de Adeboye, Andronikos, Martindale y Westbury (2016), identifica a la familia, en relación a la escuela, como una barrera para el desarrollo del talento deportivo, ya que muchas familias colocaron obstáculos contra la participación en el desarrollo deportivo y del talento, en lugar de ser una fuente de apoyo y estímulo. Los padres no autorizaron a sus hijos/as en las oportunidades de desarrollar carreras deportivas por el temor de que tal compromiso pueda reducir el rendimiento educativo.

En un estudio de caso de las divisiones inferiores de un prestigioso club de fútbol noruego, Aalberg y Sæther (2016), reportan que el club asume el desafío de la coordinación entre el deporte y la escuela. El entrenador 
jefe también tiene un puesto de coordinador en la escuela colaboradora. En la práctica esto significa que los jugadores tienen Educación Física, actividades de aprendizaje y clases de deportes de élite especializadas mientras están entrenando y el entrenador es responsable de la calificación al final del semestre. El club también ofrece el transporte ida y vuelta de la escuela a las sesiones de entrenamiento.

Según Henriksen (2010), la coordinación entre el deporte y la escuela ha demostrado ser crucial tanto para el jugador individual como para la capacidad del entorno para desarrollar deportistas exitosos. En la experiencia noruega reportada, la relación entre el club y la escuela se encuentra tan estrechamente coordinada que parece extraño ver a estas dos como instituciones competidoras. La educación y el campo deportivo a menudo están en una relación competitiva con los deportistas que quieren ser exitosos; y los facilitadores de este club de fútbol, referenciado en este estudio, parecen ser conscientes de ello (Aalberg \& Sæther, 2016).

Sin embargo, en relación con la eximición en las clases de Educación Física, Rodríguez de La Cruz (2016), manifiesta una posición crítica. El autor realiza una serie de argumentaciones por las que se concluye que, aunque estos alumnos puedan constituir la élite de una única disciplina deportiva, la exención no encaja con lo propugnado en las leyes que desarrollan el currículum español referente a la Educación Física escolar. Por todo lo cual, sostiene que quizás lo mejor fuese revisar estas medidas tan inapropiadas para una sociedad que propugna la integración y la inclusión.

Sin embargo, existen otras perspectivas que adhieren a la idea según la cual un tratamiento satisfactorio y particular a los estudiantes con potencial de excelencia deportiva, se está convirtiendo cada vez más en un emprendimiento importante y socialmente deseable. Las escuelas y las familias en este contexto se constituyen en actores claves para acompañar el desarrollo de la experiencia deportiva; sobre todo teniendo en cuenta que la edad escolar es el período más favorable para la detección, identificación, entrenamiento y desarrollo del potencial de los alumnos/as con talento deportivo (Cotê, 1999; Li \& Wang, 2014; Vidosavljević \& KruljDrasković, 2013).

En el marco de estos antecedentes es que cabe conjeturar que en la Argentina la mayoría de los talentos deportivos inician su proceso de especialización en clubes y que el espacio escolar está lejos de ser el espacio de detección y desarrollo de dichos talentos. Probablemente, existan comunidades educativas que sean especialmente sensibles al desarrollo del talento deportivo y creen las condiciones para su promoción y acompañamiento, pero ello no se constituye en un fenómeno normalizado y regulado por el sistema educativo en la órbita local ni nacional. Muy probablemente, ello se asocie a atributos de tipo individuales como el sexo. Las adolescentes mujeres de modo creciente logran mejores calificaciones escolares que sus pares varones y mejor terminalidad educativa de la escuela secundaria (Cervini \& Dari, 2009; Miranda, 2010; Tuñón \& Di Paolo, 2018), y en tal sentido probablemente logren mayores beneficios y apoyos de sus trayectorias deportivas que sus pares varones.

Asimismo, se supone que el vínculo entre el desarrollo de la especialización deportiva y la escuela guarda relación con el perfil del deportista que realiza un deporte de tipo individual o colectivo. Al respecto, Arias Estero (2008), recomienda para futuras investigaciones en el área de la especialización deportiva, que al igual que existen diferencias entre deportes individuales y colectivos, en cuanto a importancia dada a diferentes aspectos del entrenamiento, se debe analizar si los resultados de estudios que se realizan en áreas más específicas y/o en los deportes individuales son aplicables a deportes colectivos. El fin es demostrar la aplicabilidad de la información producida para el deporte en general.

Por último, se propone explorar en diferencias socio-residenciales. El desarrollo del talento deportivo en el país probablemente esté fuertemente centralizado en la Ciudad de Buenos Aires (CABA), y ello repercuta en una normativa más clara en el sistema educativo local que redunda en una situación de ventaja para sus deportistas de elite frente a los del resto del país. 
A partir de estas conjeturas se propone identificar barreras y facilitadores en relación con la escuela secundaria que han experimentado los deportistas juveniles argentinos con potencial para alcanzar la pericia deportiva.

\section{Metodología}

Se trata de un estudio no experimental, transversal y descriptivo comparativo sobre una muestra de 408 sujetos de 14 a 17 años de edad (249 varones y 159 mujeres) de un total de 530 atletas juveniles provenientes de las 24 provincias de Argentina y de 29 deportes preseleccionados para participar de los Juegos Olímpicos de la Juventud en Buenos Aires en 2018.

El instrumento utilizado toma algunos indicadores de la Encuesta para Atletas de Elite (Sport Northern Ireland, 2012) en aspectos relacionados con el desarrollo del deporte en el espacio escolar. Más específicamente, la encuesta utilizada incorpora indicadores orientados a medir el clima deportivo de elite (apoyo económico, participación política, desarrollo de talentos, carrera deportiva, instalaciones, entrenadores, competencias, investigación científica), el deporte competitivo practicado y la escuela.

El cuestionario se aplicó en la modalidad autoadministrado y se trabajó en grupos de deportistas con la asistencia de coordinadores de encuesta disponibles para evacuar dudas. La administración del instrumento se realizó entre el jueves 2 al domingo 12 de febrero de 2017 en las instalaciones del Centro Nacional de Alto Rendimiento Deportivo (CeNARD) durante el llamado Campus 2018 organizado por el Ente Nacional de Alto Rendimiento Deportivo (ENARD).

Con respecto al tratamiento de los datos se obtuvieron las frecuencias porcentuales de las respuestas de cada individuo en cada variable educativa estudiada. Estas variables fueron: espacio donde comenzó a desarrollar su deporte (escuela, club, otro); lugar donde practica su deporte (escuela, club, otro); asistencia a la escuela (si, no); práctica de deporte durante la clase de Educación Física (siempre, antes, nunca); participación en competencias deportivas escolares durante la escuela secundaria (alta, media, baja, nula); reconocimiento escolar por eximición a clases de Educación Física, por evaluación flexible, por tutorías individuales, por otros beneficios (si, no); reconocimiento mediante el apoyo de la institución escolar a la que asiste (bueno, suficiente, razonable, insuficiente, pobre, sin apoyo). Además se testearon tres afirmaciones referidas a la relación entre las sesiones de entrenamiento y ciertas obligaciones escolares: "El cansancio en los entrenamientos afecta mi rendimiento escolar"; "Dejo de lado la tarea de la escuela por el descanso previo (siesta) al entrenamiento"; "Prefiero entrenar antes que estar en la escuela estudiando" (muy alta, alta, baja, muy baja, no sabe); y una última afirmación que aludió a la continuación de los estudios luego de finalizado el nivel secundario (si, no, no sabe).

Se realizaron contrastes estadísticos mediante los tests U de Mann-Whitney y Kruskal-Wallis para detectar diferencias significativas entre grupos según sexo (masculino, femenino), tipo de deporte practicado (individual, de conjunto) y lugar de residencia (Ciudad Autónoma de Buenos Aires, Provincia de Buenos Aires, interior del país).

En todos los casos la significación estadística se estableció para $\mathrm{p}<0.05$. El tratamiento estadístico fue realizado con el software IBM SPSS Statistics versión 23.0 (IBM Corp., Armor, New York).

\section{Resultados}

En primer lugar, cabe recordar que se trata de una muestra de 408 atletas juveniles de entre 14 y 17 años preseleccionados para participar de los Juegos Olímpicos de la Juventud en Buenos Aires en 2018. Esta población en un $97,3 \%$ asistía a la escuela al momento de la encuesta. 


\section{Sobre la iniciación deportiva y la práctica del deporte}

Espacio de iniciación en el deporte:

Con relativa independencia del sexo, el tipo de deporte practicado y el lugar de residencia, en todos los casos, se observa el rol protagónico que asumen los clubes, como el principal espacio de iniciación en el deporte experto. En segundo lugar, pero con una incidencia mucho menor aparecen otros espacios como centros deportivos municipales, provinciales o nacionales, y otras organizaciones. Mientras que el espacio escolar como lugar de iniciación se registra en apenas $6,7 \%$ de la población encuestada (tabla 1 ).

\section{Lugar donde practica actualmente el deporte:}

En este caso, también se advierte que el espacio del club es el principal lugar de práctica del deporte en el proceso de especialización $(79,2 \%)$ (tabla 1 ). No obstante, es interesante señalar que en el caso del deporte colectivo se registra una propensión por encima del promedio, a iniciar el deporte en el espacio escolar, mientras que el deporte individual en otros espacios. Asimismo, cuando se considera el lugar de práctica en relación al lugar de residencia, se constata que la práctica en otros espacios representa en Provincia de Buenos Aires el 20,6\%, y la práctica en escuelas de la Ciudad Autónoma de Buenos Aires (CABA), el 14,6\%, revelándose diferencias significativas $(\mathrm{H}=6,466 ; \mathrm{p}=0,039)$, como se observa en la tabla 6 .

Tabla 1. Frecuencias porcentuales para el espacio donde inicia el deporte practicado y lugar actual donde practica su deporte, según sexo, tipo de deporte practicado y lugar de residencia

\begin{tabular}{|c|c|c|c|c|c|c|c|}
\hline \multirow{2}{*}{\multicolumn{2}{|c|}{$\begin{array}{l}\text { FORMACION } \\
\text { DEPORTIVA }\end{array}$}} & \multicolumn{3}{|c|}{$\begin{array}{l}\text { Es pacio donde } \\
\text { comienza su deporte }\end{array}$} & \multicolumn{3}{|c|}{ Lugar donde practica su deporte } \\
\hline & & Escuela & Club & Otro & Escuela & Club & Otro \\
\hline \multirow{2}{*}{ Sexo } & Masculino & $6,05 \%$ & $79,03 \%$ & $14,92 \%$ & $6,0 \%$ & $79,0 \%$ & $14,9 \%$ \\
\hline & Femenino & $7,6 \%$ & $81,0 \%$ & $11,4 \%$ & $5,9 \%$ & $80,6 \%$ & $13,5 \%$ \\
\hline \multirow{2}{*}{$\begin{array}{l}\text { Deporte } \\
\text { practicado }\end{array}$} & Individual & $7,6 \%$ & $76,2 \%$ & $16,2 \%$ & $3,3 \%$ & $77,9 \%$ & $18,8 \%$ \\
\hline & Colectivo & $4,8 \%$ & $87,1 \%$ & $8,1 \%$ & $12,1 \%$ & $83,9 \%$ & $4,0 \%$ \\
\hline \multirow{3}{*}{$\begin{array}{l}\text { Lugar de } \\
\text { residencia }\end{array}$} & C.A.B.A. & $8,3 \%$ & $85,4 \%$ & $6,3 \%$ & $14,6 \%$ & $77,1 \%$ & $8,3 \%$ \\
\hline & Bs. As. & $5,0 \%$ & $78,1 \%$ & $16,9 \%$ & $5,6 \%$ & $73,8 \%$ & $20,6 \%$ \\
\hline & Interior & $7,7 \%$ & $79,6 \%$ & $12,8 \%$ & $4,6 \%$ & $84,1 \%$ & $11,3 \%$ \\
\hline \multicolumn{2}{|c|}{ TOTAL } & $6,7 \%$ & $79,7 \%$ & $13,6 \%$ & $6,2 \%$ & $79,2 \%$ & $14,6 \%$ \\
\hline
\end{tabular}

\section{Práctica de deporte durante la clase de Educación Física:}

Tal como se registra en tabla 2, la gran mayoría de los encuestados practica un deporte en las clases de educación física escolar (82,6\%). Sin embargo, ello es mucho más probable que ocurra entre quienes se entrenan en un deporte colectivo $(94,9 \%$, frente a $76 \%$ en quienes realizan un deporte individual) $(\mathrm{U}=$ $10,937 ; \mathrm{p}=0,001)$. 


\section{Participación en competencias deportivas escolares durante la escuela secundaria:}

La mayoría de los adolescentes consultados reportan no participar nunca o pocas veces de competencias deportivas escolares (36,7\% y 23,6\%, respectivamente). Es relevante señalar que es más probable no tener ocasión para participar de estas competencias si se realiza un deporte de tipo individual. Por otro lado, se registra que casi 4 de cada 10 participan de competencias deportivas muchas veces o algunas veces (23,4\% y $16,3 \%$, respectivamente). Quienes practican deportes de conjunto tienen más probabilidad de participar de competencias deportivas escolares que quienes realizan deportes individuales ( $42,4 \%$ y $24,2 \%$, respectivamente) $(\mathrm{U}=12,849 ; \mathrm{p}=0,02$; ver tabla 6$)$.

Tabla 2. Frecuencias porcentuales para práctica deportiva en clase de Educación Física y participación en competencias escolares en la escuela secundaria, según sexo, tipo de deporte practicado y lugar de residencia

\begin{tabular}{|c|c|c|c|c|c|c|c|c|}
\hline \multirow{2}{*}{\multicolumn{2}{|c|}{ DEPORTE ESCOLAR }} & \multicolumn{3}{|c|}{$\begin{array}{l}\text { Práctica de deporte durante la } \\
\text { clase de educación fisica }\end{array}$} & \multicolumn{4}{|c|}{$\begin{array}{l}\text { Participación en competencias deportivas } \\
\text { escolares dura nte la escuela secundaria }\end{array}$} \\
\hline & & Siempre & Antes & Nunca & $\begin{array}{c}\text { Muchas } \\
\text { veces }\end{array}$ & A veces & $\begin{array}{l}\text { Pocas } \\
\text { veces }\end{array}$ & Nunca \\
\hline \multirow{2}{*}{ Sexo } & Mas culino & $83,1 \%$ & $5,9 \%$ & $11,0 \%$ & $24,0 \%$ & $17,1 \%$ & $23,6 \%$ & $35,4 \%$ \\
\hline & Femenino & $81,5 \%$ & $10,3 \%$ & $8,2 \%$ & $22,1 \%$ & $16,2 \%$ & $24,0 \%$ & $37,7 \%$ \\
\hline \multirow{2}{*}{$\begin{array}{c}\text { Deporte } \\
\text { practica do }\end{array}$} & Individual & $76,0 \%$ & $9,9 \%$ & $14,0 \%$ & $19,6 \%$ & $16,6 \%$ & $21,4 \%$ & $42,4 \%$ \\
\hline & Colec tivo & $94,9 \%$ & $3,4 \%$ & $1,7 \%$ & $30,6 \%$ & $16,9 \%$ & $28,2 \%$ & $24,2 \%$ \\
\hline \multirow{3}{*}{$\begin{array}{l}\text { Lugar de } \\
\text { residencia }\end{array}$} & C.A.B.A. & $81,0 \%$ & $7,1 \%$ & $11,9 \%$ & $26,1 \%$ & $17,4 \%$ & $15,2 \%$ & $41,3 \%$ \\
\hline & Bs. As. & $79,1 \%$ & $8,1 \%$ & $12,8 \%$ & $21,4 \%$ & $18,2 \%$ & $20,1 \%$ & $40,3 \%$ \\
\hline & Interior & $86,1 \%$ & $7,5 \%$ & $6,4 \%$ & $24,4 \%$ & $14,5 \%$ & $28,5 \%$ & $32,6 \%$ \\
\hline \multicolumn{2}{|c|}{ TOTAL } & $82,6 \%$ & $7,7 \%$ & $9,6 \%$ & $23,4 \%$ & $16,3 \%$ & $23,6 \%$ & $36,7 \%$ \\
\hline
\end{tabular}

\section{Sobre el reconocimiento de la práctica del deporte en el espacio escolar}

\section{Eximición de clases de Educación Física:}

Tanto por sexo como según el tipo de deporte practicado, la propensión a no ser eximido de la clase de educación física fluctúa en un rango de 31,8\% a 37,9\% de los encuestados, existiendo una diferencia de 6,1 puntos porcentuales en perjuicio de los que practican deportes individuales, respecto de quienes practican deportes de conjunto.

En cuanto a la relación con las jurisdicciones de residencia, se observa que en el interior del país (43,2\%) hay menos reconocimiento a la eximición que en Provincia de Buenos Aires (33,2\%); siendo la CABA la más favorecida (sólo 18,9\% de no eximición) (ver tabla 3), revelándose aquí diferencias significativas $(\mathrm{H}=6,169$; $\mathrm{p}=0,046$; ver tabla 6).

\section{Flexibilidad en la toma de evaluaciones:}

Al considerar las diferencias por sexo, la falta de reconocimiento fue 6,9 puntos porcentuales (p.p.) superior en varones que, en mujeres, y entre 18,7 p.p. y 15,5 p.p. superior en CABA, en comparación con Provincia de Buenos Aires y el Interior del país, respectivamente (ver tabla 3). Las diferencias observadas entre jurisdicciones también fueron significativas, como se expone en la tabla $6(\mathrm{H}=6,668 ; \mathrm{p}=0,036)$. 


\section{Asesoramiento individual (tutorias) en contenidos dictados:}

Se explicita en tabla 3 que, en relación con el sexo, el deporte practicado y la jurisdicción de residencia, la falta de reconocimiento es elevado, ubicándose entre el $67,2 \%$ y el 78,8\% de los encuestados. No obstante, se estima que un $27,2 \%$ recibe tutorías y ello es más probable entre las mujeres que entre los varones, y en el interior del país más que en la CABA y Provincia de Buenos Aires.

Otros beneficios (cambios de turno, entrenamientos por las mañanas, eximición a talleres, llegadas tarde, entre los más importantes):

La gran mayoría de los encuestados reportan no contar con este tipo de beneficios (86,7\%). No obstante, la propensión a tener estos beneficios es mucho mayor entre las mujeres y entre los residentes en el interior del país que en Provincia de Buenos Aires y la CABA (ver tabla 3).

Tabla 3. Frecuencias porcentuales para distintos reconocimientos escolares a la práctica deportiva federada, según sexo, tipo de deporte practicado y lugar de residencia

\begin{tabular}{|c|c|c|c|c|c|c|c|c|c|}
\hline \multirow{2}{*}{\multicolumn{2}{|c|}{$\begin{array}{l}\text { RECONOCIMIENTO } \\
\text { DEORTIVOESCOLAR }\end{array}$}} & \multicolumn{2}{|c|}{\begin{tabular}{|} 
Reconocimiento \\
escolar por eximición a \\
clases de Educación \\
Fisica
\end{tabular}} & \multicolumn{2}{|c|}{$\begin{array}{l}\text { Reconocimiento } \\
\text { escolar por flexibilidad } \\
\text { en la toma de } \\
\text { evaluaciones }\end{array}$} & \multicolumn{2}{|c|}{$\begin{array}{l}\text { Reconocimiento } \\
\text { escolar por } \\
\text { asesoramiento } \\
\text { individual (tutorías) en } \\
\text { contenidos dictados }\end{array}$} & \multicolumn{2}{|c|}{\begin{tabular}{|} 
Reconocimiento \\
escolar por otros \\
beneficios (cambios de \\
turno, entrenamientos \\
por las mañanas, \\
eximición a talleres, \\
llegadas tarde, entre \\
los más importantes)
\end{tabular}} \\
\hline & & si & No & Si & No & si & No & si & No \\
\hline \multirow{2}{*}{ Sexo } & Masculino & $64,4 \%$ & $35,6 \%$ & $73,9 \%$ & $26,1 \%$ & $25,3 \%$ & $74,7 \%$ & $7,1 \%$ & $92,9 \%$ \\
\hline & Femenino & $63,3 \%$ & $36,7 \%$ & $80,8 \%$ & $19,2 \%$ & $30,2 \%$ & $69,8 \%$ & $25,7 \%$ & $74,3 \%$ \\
\hline \multirow{2}{*}{$\begin{array}{c}\text { Deporte } \\
\text { practicado }\end{array}$} & Individual & $62,1 \%$ & 37,996 & $75,9 \%$ & $24,1 \%$ & $27,9 \%$ & $72,1 \%$ & $13,2 \%$ & $86,8 \%$ \\
\hline & Colectivo & $68,2 \%$ & $31,8 \%$ & $77,6 \%$ & $22,4 \%$ & $26,5 \%$ & $73,5 \%$ & $15,4 \%$ & $84,6 \%$ \\
\hline \multirow{3}{*}{$\begin{array}{l}\text { Lugar de } \\
\text { residencia }\end{array}$} & C.A.B.A. & $81,1 \%$ & $18,9 \%$ & $61,1 \%$ & $38,9 \%$ & $25,7 \%$ & $74,3 \%$ & $6,7 \%$ & $93,3 \%$ \\
\hline & Bs. As. & $68,1 \%$ & $33,9 \%$ & $79,8 \%$ & $20,2 \%$ & $21,2 \%$ & $78,8 \%$ & $6,7 \%$ & $93,3 \%$ \\
\hline & Interior & $58,8 \%$ & $43,2 \%$ & $78,6 \%$ & $23,4 \%$ & $32,8 \%$ & $67,2 \%$ & $22,2 \%$ & $77,8 \%$ \\
\hline \multicolumn{2}{|c|}{ TOTAL } & $63,6 \%$ & $36,4 \%$ & $78,0 \%$ & $24,0 \%$ & $27,2 \%$ & $72,8 \%$ & $13,3 \%$ & $86,7 \%$ \\
\hline
\end{tabular}

\section{Evaluación del apoyo recibido de la institución escolar a la que asiste:}

El 71,3\% de los encuestados reporta que el reconocimiento es bueno, suficiente o razonable, mientras que el restante 28,6\% expresó que el apoyo es insuficiente, pobre o nulo. No se registran diferencias significativas según el sexo, y el tipo de deporte practicado, pero en el caso de los residentes de la CABA la probabilidad de contar con apoyo es mayor que entre pares que residen en otros lugares. 
Tabla 4. Frecuencias porcentuales para el apoyo de la institución escolar a la que asiste a la práctica deportiva federada, según sexo, tipo de deporte practicado y lugar de residencia

\begin{tabular}{|c|c|c|c|c|c|c|c|}
\hline \multirow{2}{*}{\multicolumn{2}{|c|}{$\begin{array}{c}\text { RECONOCIMIENTO } \\
\text { DEPORTIVO ESCOLAR }\end{array}$}} & \multicolumn{6}{|c|}{$\begin{array}{l}\text { Reconocimiento mediante el apoyo de la institución escolar a la que } \\
\text { asiste }\end{array}$} \\
\hline & & Bueno & Suficiente & Razonable & Insuficiente & Pobre & Sin apoyo \\
\hline \multirow{2}{*}{ Sexo } & Masculino & $27,4 \%$ & $21,0 \%$ & $23,3 \%$ & $9,1 \%$ & $6,4 \%$ & $12,8 \%$ \\
\hline & Femenino & $30,3 \%$ & $15,9 \%$ & $25,5 \%$ & $8,3 \%$ & $9,7 \%$ & $10,3 \%$ \\
\hline \multirow{2}{*}{$\begin{array}{c}\text { Deporte } \\
\text { practicado }\end{array}$} & Individual & $31,5 \%$ & $18,5 \%$ & $21,8 \%$ & $8,1 \%$ & $7,3 \%$ & $12,9 \%$ \\
\hline & Colectivo & $22,3 \%$ & $19,6 \%$ & $30,4 \%$ & $10,7 \%$ & $8,0 \%$ & $8,9 \%$ \\
\hline \multirow{3}{*}{$\begin{array}{l}\text { Lugar de } \\
\text { residencia }\end{array}$} & C.A.B.A. & $39,5 \%$ & $20,9 \%$ & $14,0 \%$ & $7,0 \%$ & $2,3 \%$ & $16,3 \%$ \\
\hline & Bs. As. & $29,7 \%$ & $12,2 \%$ & $28,4 \%$ & $8,8 \%$ & $7,4 \%$ & $13,5 \%$ \\
\hline & Interior & $24,4 \%$ & $23,8 \%$ & $23,3 \%$ & $9,3 \%$ & $9,3 \%$ & $9,9 \%$ \\
\hline \multicolumn{2}{|c|}{ TOTAL } & $28,4 \%$ & $18,7 \%$ & $24,2 \%$ & $8,8 \%$ & $7,7 \%$ & $12,1 \%$ \\
\hline
\end{tabular}

\section{Representaciones en torno al vínculo entre obligaciones escolares y práctica del deporte}

A continuación, se analiza la evaluación que realizan los encuestados en relación con un conjunto de afirmaciones que procuran representar la relación entre las sesiones de entrenamiento y las obligaciones escolares. Frente a cada una de las afirmaciones los adolescentes consultados respondieron con una escala de frecuencia de percepción de la ocurrencia de dichas situaciones. Finalmente, una última afirmación refirió acerca del deseo de continuar estudios luego de finalizar la educación secundaria.

Los resultados, pueden observarse en la tabla 5: 
Tabla 5. Frecuencias porcentuales para las percepciones sobre la relación entre el deporte y la escuela, según sexo, tipo de deporte practicado y lugar de residencia

\begin{tabular}{|c|c|c|c|c|c|c|c|c|c|}
\hline \multirow{2}{*}{\multicolumn{2}{|c|}{$\begin{array}{c}\text { DEPORTE Y } \\
\text { ESCUELA }\end{array}$}} & \multirow{2}{*}{\multicolumn{2}{|c|}{\begin{tabular}{|c|} 
Sexo \\
Mas culino Femenino
\end{tabular}}} & \multicolumn{2}{|c|}{ Deporte practicado } & \multicolumn{3}{|c|}{ Luga r de residencia } & \multirow{2}{*}{ TOTAL } \\
\hline & & & & Individual & Colectivo & C.A.B.A. & Bs. As. & Interior & \\
\hline \multirow{5}{*}{ 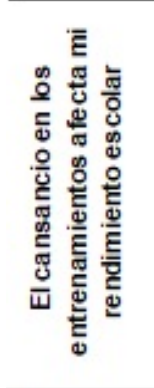 } & $\begin{array}{c}\text { Cas } i \\
\text { s iempre }\end{array}$ & $5,7 \%$ & $5,1 \%$ & $4,8 \%$ & $5,7 \%$ & $6,4 \%$ & $5,7 \%$ & $4,7 \%$ & $5,3 \%$ \\
\hline & $\begin{array}{c}\text { Muchas } \\
\text { veces }\end{array}$ & $20,5 \%$ & $21,2 \%$ & $19,2 \%$ & $23,6 \%$ & $17,0 \%$ & $24,8 \%$ & $18,7 \%$ & $20,9 \%$ \\
\hline & $\begin{array}{l}\text { Pocas } \\
\text { veces }\end{array}$ & $34,8 \%$ & $30,1 \%$ & $32,8 \%$ & $35,0 \%$ & $27,7 \%$ & $31,2 \%$ & $36,3 \%$ & $33,2 \%$ \\
\hline & $\begin{array}{c}\text { Cas } i \\
\text { nunca }\end{array}$ & $34,4 \%$ & $42,3 \%$ & $39,9 \%$ & $32,5 \%$ & $46,8 \%$ & $35,0 \%$ & $36,8 \%$ & $37,3 \%$ \\
\hline & $\begin{array}{c}\text { No } \\
\text { sabe }\end{array}$ & $4,5 \%$ & $1,3 \%$ & $3,3 \%$ & $3,3 \%$ & $2,1 \%$ & $3,2 \%$ & $3,6 \%$ & $3,3 \%$ \\
\hline \multirow{5}{*}{ 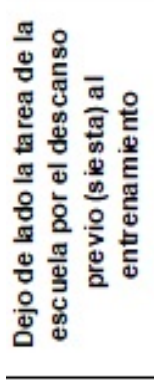 } & $\begin{array}{c}\text { Cas } i \\
\text { siempre }\end{array}$ & $18,2 \%$ & $17,9 \%$ & $16,2 \%$ & $20,7 \%$ & $17,0 \%$ & $21,7 \%$ & $15,2 \%$ & $18,0 \%$ \\
\hline & $\begin{array}{c}\text { Muchas } \\
\text { veces }\end{array}$ & $23,6 \%$ & $19,2 \%$ & $19,9 \%$ & $27,3 \%$ & $21,3 \%$ & $21,7 \%$ & $22,0 \%$ & $21,8 \%$ \\
\hline & $\begin{array}{l}\text { Pocas } \\
\text { veces }\end{array}$ & $27,7 \%$ & $28,8 \%$ & $30,3 \%$ & $24,8 \%$ & $27,7 \%$ & $31,8 \%$ & $25,1 \%$ & $28,1 \%$ \\
\hline & $\begin{array}{c}\text { Cas } i \\
\text { nunca }\end{array}$ & $26,9 \%$ & $33,3 \%$ & $31,4 \%$ & $24,8 \%$ & $34,0 \%$ & $22,3 \%$ & $35,1 \%$ & $29,9 \%$ \\
\hline & $\begin{array}{c}\text { No } \\
\text { sabe }\end{array}$ & $3,7 \%$ &, $6 \%$ & $2,2 \%$ & $2,5 \%$ & $0 \%$ & $2,5 \%$ & $2,6 \%$ & $2,3 \%$ \\
\hline \multirow{5}{*}{ 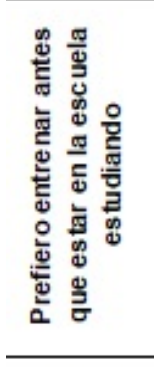 } & $\begin{array}{c}\text { Cas } i \\
\text { siempre }\end{array}$ & $60,0 \%$ & $48,7 \%$ & $51,1 \%$ & $64,2 \%$ & $58,3 \%$ & $59,5 \%$ & $50,0 \%$ & $54,8 \%$ \\
\hline & $\begin{array}{c}\text { Muchas } \\
\text { veces }\end{array}$ & $23,3 \%$ & $32,1 \%$ & $28,8 \%$ & $22,0 \%$ & $33,3 \%$ & $23,4 \%$ & $28,7 \%$ & $27,0 \%$ \\
\hline & $\begin{array}{l}\text { Pocas } \\
\text { veces }\end{array}$ & $8,9 \%$ & $10,9 \%$ & $11,7 \%$ & $4,9 \%$ & $4,2 \%$ & $10,8 \%$ & $10,5 \%$ & $9,9 \%$ \\
\hline & $\begin{array}{c}\text { Cas } i \\
\text { nunca }\end{array}$ & $5,8 \%$ & $5,8 \%$ & $6,2 \%$ & $5,7 \%$ & $4,2 \%$ & $4,4 \%$ & $7,7 \%$ & $6,1 \%$ \\
\hline & $\begin{array}{c}\text { No } \\
\text { sabe }\end{array}$ & $2,0 \%$ & $2,6 \%$ & $1,8 \%$ & $3,3 \%$ & $0 \%$ & $1,9 \%$ & $3,1 \%$ & $2,3 \%$ \\
\hline \multirow{3}{*}{ 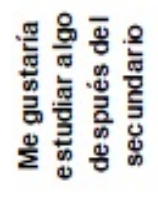 } & Si & $82,9 \%$ & $96,8 \%$ & $88,3 \%$ & $88,7 \%$ & $91,7 \%$ & $86,9 \%$ & $89,1 \%$ & $88,5 \%$ \\
\hline & No & $7,8 \%$ &, $6 \%$ & $5,5 \%$ & $4,0 \%$ & $4,2 \%$ & $6,3 \%$ & $4,1 \%$ & $5,0 \%$ \\
\hline & No sabe & $9,4 \%$ & $2,5 \%$ & $6,2 \%$ & $7,3 \%$ & $4,2 \%$ & $6,9 \%$ & $6,7 \%$ & $6,5 \%$ \\
\hline
\end{tabular}

\section{"El cansancio de los entrenamientos afecta mi rendimiento escolar"}

La mayoría de los encuestados reporta que casi nunca o pocas veces el cansancio de los entrenamientos afecta su rendimiento escolar ( $37,3 \%$ y $33,2 \%$, respectivamente). No obstante, entre las mujeres esto parece ser más frecuente que entre los varones y entre quienes realizan un deporte individual que entre quienes practican deportes colectivos.

\section{"Dejo de lado la tarea de la escuela por el descanso previo (siesta) al entrenamiento"}

En este punto los reportes están divididos, por un lado, el 58\% de los encuestados expresa que pocas veces o nunca deja de lado las tareas de la escuela por el descanso; pero, por otro lado, se registra que un $40 \%$ 
expresa que esto suele suceder. Entre estos últimos se destaca la mayor propensión de los varones frente a las mujeres y de quienes practican deportes colectivos frente a los que realizan deportes individuales.

\section{"Prefiero entrenar antes que estar en la escuela estudiando"}

Ocho de cada diez encuestados adhiere a la afirmación de referencia como algo que les sucede casi siempre y muchas veces $(54,8 \%$ y $27 \%$, respectivamente). No obstante, se advierte que los adolescentes varones adhieren en mayor medida que sus pares mujeres a la afirmación de referencia, así como quienes practican deportes colectivos que quienes realizan deportes individuales, y se destaca la mayor adhesión de los residentes en la CABA frente a los residentes en otros lugares del país.

\section{"Me gustaría estudiar algo después del secundario"}

La gran mayoría de los encuestados adhiere a la afirmación de referencia (88,5\%). Sin embargo, se registran diferencias significativas entre sexos a favor de las mujeres ( $96,8 \%$ mujeres y $82,9 \%$ varones $)(\mathrm{U}=$ $16,676 ; \mathrm{p}=0,001)$.

Tabla 6. Variables dependientes con diferencias significativas para las pruebas U de Mann-

Whitney y $\mathrm{H}$ de Kruskal Wallis, según sexo, tipo de deporte practicado y lugar de residencia

\begin{tabular}{c|ccc} 
& U & H & P \\
\hline Lugar de práctica de su deporte & & 6,468 & 0,039 \\
Práctica deportiva en clase de Educación Física & 10,937 & & 0,001 \\
$\begin{array}{c}\text { Participación en competencias deportivas } \\
\text { escolares }\end{array}$ & 12,849 & 0,02 \\
$\begin{array}{c}\text { Reconocimiento escolar: eximición clase de } \\
\text { Educación Fisica }\end{array}$ & 6,169 & 0,046 \\
$\begin{array}{c}\text { Reconocimiento escolar: flexibilidad en toma de } \\
\text { evaluaciones }\end{array}$ & 6,668 & 0,036 \\
$\begin{array}{c}\text { Continuación de los estudios luego del } \\
\text { secundario }\end{array}$ & 16,676 & & 0,001
\end{tabular}

\section{Discusión}

Los resultados hasta aquí expuestos indican con mucha claridad que el club es el principal espacio de iniciación y especialización deportiva del país, y que la escuela cumple un rol muy inferior en este sentido. Ahora, la relación del deportista con el espacio escolar en el proceso de desarrollo de su talento puede calificarse positivamente. Es decir, la mayoría de los encuestados reportaron tener beneficios (apoyo) en la eximición de las clases de educación física y consideración en las evaluaciones. Otros beneficios como las tutorías respecto a los contenidos dictados, son muy inferiores en su incidencia, y parecen estar más asociados a estrategias de tipo individuales, mejor desarrolladas por las adolescentes mujeres que por sus pares varones. Inicialmente se conjeturó que ello podría estar vinculado al mejor rendimiento educativo que en promedio tienen las mujeres, y otras habilidades blandas que se podrían explorar en estudios futuros.

En referencia a la influencia de los espacios club y escuela respecto a la iniciación y especialización deportiva, Adeboye, Andronikos, Martindale y Westbury (2016) reportan para el caso de Nigeria que los clubes no brindan suficientes oportunidades para competir. Esta carencia se ve favorecida por la mala organización y administración del deporte en este país, donde no existe una estructura de club que permita a los atletas 
de estas diferentes instituciones competir entre ellos para lograr un rendimiento de referencia. Si bien no es el caso de la Argentina, donde el club es el principal espacio de especialización, es interesante la relación que establecen entre el deporte y la escuela. En este caso, la escuela ofrece una oportunidad para el desarrollo de talentos a través de la competición deportiva anual Inter-House, tradición en la mayoría de las escuelas nigerianas. Todos los participantes informaron que participaron en el deporte escolar y que esa participación formó la base para su eventual desarrollo. Para los atletas entrevistados, la escuela es el único lugar en el que había alguna apariencia de deporte organizado disponible. Cabe destacar que, en Argentina, estas competencias Inter-House, no sólo en deportes, sino también en conocimientos generales, música y otras áreas, son características de diversos colegios privados bilingües, especialmente de comunidades inglesas y escocesas, entre otras; aunque se encuentran reservadas a niños/as y adolescentes de elevado estrato socioeconómico.

Cabe advertir que casi un tercio de los deportistas reportaron un apoyo deficitario por parte de la institución educativa a la que asisten y ello se constituye en un dato relevante en sí mismo. Por otro lado, también se registran carencias respecto a los deportes individuales, tanto en la práctica de las clases de educación física, como en la oferta de competencias deportivas escolares.

Respecto de las percepciones en relación con la incidencia del entrenamiento en las obligaciones escolares y en la continuación de los estudios, surge que hay un porcentaje elevado de alumnos deportistas que refieren ciertas dificultades tanto en el rendimiento escolar como en el cumplimiento de obligaciones asociadas al mismo como consecuencia de los procesos de entrenamiento deportivo. Además, hay una marcada tendencia a la preferencia por la práctica deportiva respecto a la permanencia en la escuela ante su tarea formativa. Sin embargo, a la mayoría de los deportistas le gustaría continuar con sus estudios al finalizar la escuela secundaria. Es decir, que no parece existir un desinterés por la formación escolar.

En relación a lo anterior y respecto a los reconocimientos escolares en cuanto a eximición a clases de Educación Física, flexibilidad en la toma de evaluaciones y asesoramiento individual (tutorías) en contenidos dictados, los resultados indican que los alumnos que asisten a escuelas del Gobierno de la Ciudad de Buenos Aires (GCBA) son los más beneficiados. Esto guardaría coherencia con la ley 5823, sancionada por la Legislatura de la CABA, donde se establecen estos beneficios para alumnos de nivel secundario que asisten a todas las escuelas dependientes del GCBA. Aunque, respecto a otros beneficios como cambios de turno, entrenamientos por las mañanas, eximición a talleres, llegadas tarde, entre los más importantes, los alumnos más beneficiados fueron los del interior del país, aunque las diferencias no son estadísticamente significativas.

Si bien, como resulta de este estudio, las situaciones de déficits de las variables educativas asociadas al proceso de formación del talento deportivo juvenil, están lejos de ser extendidas, pero afectan a aproximadamente un tercio de la población encuestada. En este sentido, parece relevante presentar los argumentos de una perspectiva orientada hacia el interior de la comunidad educativa. Desde este enfoque la escuela podría promover una relación con estudiantes talentosos en el deporte que no sólo se reduzca la resolución de casos individuales, sino que desarrolle un sistema de medidas para regular el status de esta categoría de alumnos. La evolución de estos talentos, se conjetura que puede depender de manera importante del compromiso de los docentes en general y profesores de Educación Física en particular. Es decir, que el reconocimiento de habilidades y la identificación de jóvenes talentosos en los deportes, el conocimiento de sus habilidades, el trabajo metódico eficaz con estos estudiantes, puede lograr buenas formas de organización y metodologías adecuadas vinculadas a la formación (Olovacha Toroshina, 2015; Medranda Rojas, Castillejo, Pérez Ramírez \& Alonso Betancourt, 2017).

Existe consenso en torno a que el espacio escolar y familiar es relevante que se constituyan en entornos de apoyo estimulante y enriquecido para un mejor desarrollo del potencial. Propiciar una buena comunicación entre las familias y las escuelas es algo recomendado para asegurar la integridad y sustentabilidad de los efectos educativos en estos individuos. Los grupos interesados, ya sea personas físicas, instituciones u organizaciones (stakeholders) asociados al desarrollo de estos talentos deportivos juveniles, la familia y las escuelas, se 
recomienda establezcan fuertes alianzas que estimulen el crecimiento, la educación y el desarrollo óptimo de estos jóvenes talentosos en las más diversas actividades deportivas (Cotê, 1999; Li \& Wang, 2014; Vidosavljević \& Krulj-Drasković, 2013). La literatura especializada sugiere direccionar más intensamente las acciones con los stakeholders en su vínculo con la escuela, y que ellos se conviertan en monitores y asesores para la obtención de metas regulares y realizables en la práctica cotidiana. Esto no sólo implica nuevos métodos y técnicas deportivas significativas y efectivas, sino también una revisión del rol de los profesores de Educación Física, así como una mayor cooperación constructiva y sinérgica con las familias (Vidosavljević \& KruljDrasković, 2013).

Existe consenso en torno a que la contribución de las familias, en colaboración con los docentes y la comunidad educativa en un sentido amplio, pueden coadyuvar al desarrollo integral de los individuos dotados en un contexto de mejores estructuras de oportunidades para todos (Kirk, 2005; Agudelo et al., 2016; Kay, 2000; Velásquez, 2006).

\section{Conclusiones}

El presente estudio revela varios datos muy auspiciosos en torno al rol de los clubes como espacio de detección y desarrollo de talentos deportivos. Tambin permite advertir que la relación de la escuela con los estudiantes deportistas en la mayoría de los casos es de apoyo, y que cuentan con beneficios que propician la especialización. Sin embargo, el estudio permite reconocer matices y poblaciones minoritarias que no experimentan los mismos beneficios y que se estima pueden constituirse en evidencia relevante para mejorar el rol de la escuela en relación al desarrollo del talento deportivo.

En primer lugar, cabe preguntarse, por qué la escuela no podría constituirse en un espacio de detección de talentos deportivos alternativo y complementario al club. En el marco de esta misma investigación, en otra publicación, se señaló el homogéneo perfil socioeconómico de nivel medio alto que caracteriza a los deportistas de elite (Farinola et al., 2018). Adicionalmente, se estima que el $60 \%$ de los adolescentes en el país no realiza actividad física ni deportiva estructurada extra-escolar (Tuñón, 2019). Es decir, que la mayoría de los adolescentes de la Argentina no accede al deporte en los clubes y quienes lo hacen suelen pertenecer a hogares de estratos medios altos, lo cual permite conjeturar que el espacio escolar podría asumir un rol protagónico en la identificación y seguimiento de adolescentes potenciales expertos deportivo de un universo socialmente más heterogéneo.

Existe una estrategia denominada Educación Deportiva, que es un modelo curricular de enseñanza que surge con el propósito de estimular, durante las clases de Educación Física y actividades extracurriculares, experiencias en las que los alumnos tengan las mismas oportunidades de práctica, lleguen a ser motrizmente competentes, adquieran una cultura deportiva, y se entusiasmen con la práctica. Este modelo se centra en el aprendizaje del alumno, a través de una pedagogía cooperativa y constructivista, facilitada por el trabajo en pequeños grupos y juegos reducidos en los que cada miembro tiene un rol y, en general, persigue un objetivo de mejoras técnica y conceptual, en la toma de decisiones y en el grado de responsabilidad y autonomía. Incluso, distintas investigaciones evidencian que con este modelo se alcanzan y superan las recomendaciones internacionales en cuanto a la cantidad e intensidad de actividad física realizada en la clase de Educación Física (Siedentop, 1994, Hastie \& Trost, 2002).

La otra perspectiva orientada hacia las políticas públicas, podría asegurar estrategias conjuntas desde las áreas de educación y deportes, coordinadas desde la nación hacia las provincias y municipios. Para ello, se considera como una interesante oportunidad que el Ministerio de Educación y Deportes de la Nación, que, a partir de la Secretaría de Deporte, Educación Física y Recreación, con sus correspondientes Subsecretarías de Deportes y Alto Rendimiento Deportivo; y de Educación Física, Recreación e Infraestructura, que podrían diseñar e implementar intervenciones que puedan atender a las necesidades descriptas. Evidentemente, 
también se considera que tanto el ENARD como el Comité Olímpico Argentino, podrían tener un rol protagónico en este proceso.

\section{Agradecimientos}

Este artículo se desarrolló en el marco del Proyecto PROINCE 55-A-223 “Aspectos sociodemográficos, psicológicos, familiares y físicos asociados a la expertice deportiva. El caso de los deportistas seleccionados para participar de los Juegos Olímpicos de la Juventud 2018”, del Departamento de Humanidades y Ciencias Sociales de la Universidad Nacional de La Matanza.

Se agradece los valiosos comentarios y sugerencias de los evaluadores anónimos de la Revista Educación Física y Ciencia. Asimismo, se reconoce al equipo de investigación que realizó el relevamiento de datos: Martín Farinola, Fernando Laíño, Martín Pérez Rodríguez, Hernán Castro, Brigida Lavignolle, Mario Marchesich, y Albino Rodríguez Franco, bajo la dirección de Ianina Tuñón. Un agradecimiento especial al Ente Nacional de Alto Rendimiento Deportivo (ENARD).

\section{REFERENCIAS}

Aalberg, R.R. \& Sæther, S.A. (2016). The talent development environment in a Norwegian top-level football club. Sports Science Review, 25(3/4), 159-182.

Adeboye, I. E., Andronikos, G., Martindale, R. \& Westbury, T. (2016). Talent development environment in Nigeria: athletes' perceptions of barriers, opportunities and facilitators. American Journal of Sports Science, 4(6), 98-104.

Agudelo, A., Ayala, C., Ramos, S., García, A., Melo, L., Aguirre-Loaiza, H., Martínez, K. (2016). Características de la formación deportiva de los participantes en IV Juegos Escolares Centroamericanos y del Caribe, Armenia 2013. Lúdica Pedagógica, 1(23), 127-139.

Arias Estero, J.L. (2008). El proceso de formación deportiva en la iniciación a los deportes colectivos fundamentado en las características del deportista experto. Retos. Nuevas tendencias en Educación Física, Deporte y Recreación, 13, 28-32.

Cervini, R. y Dari, N. (2009). Género, escuela y logro escolar en matemática y lengua de la educación media: estudio exploratorio basado en un modelo multinivel bivariado. RMIE, 14(42), 1051-1078.

Cotê, J. (1999). The influence of the family in the development of talent in sport. The Sport Psychologist, 13(4), 395-417.

Farinola, M. G., Tuñón, I., Laíño, F., Marchesich, M. y Pérez Rodríguez, M. (2018). Perfil socioeducativo y económico de deportistas adolescentes de élite argentinos. Retos, 34, 172-176.

Hastie, P. A. \& Trost, S. G. (2002). Student physical activity levels during a season of Sport Education. Pediatric Exercise Science, 14(1), 64-74.

Henriksen, K. (2010). The ecology of talent development in sport: a multiple case study of successful athletic talent development environments in Scandinavia (Unpublished doctoral dissertation). University of Southern Denmark, Denmark.

Kay, T. (2000). Sporting excellence: A family affair? European Physical Education Review, 6(2), 151-169.

Kirk, D. (2005). Physical education, youth sport and lifelong participation: the importance of early learning experiences. European Physical Education Review, 11(3), 239-255.

Medranda Rojas, J. L., Castillejo, O. R., Pérez Ramírez, R. M. y Alonso Betancourt, L. A. (2017). Fundamentos teóricos y metodológicos para la identificación de talentos deportivos en la educación física escolar.Didasc@lia:Didáctica y Educación, 8(1). Recuperado de https://dialnet.unirioja.es/servlet/articulo?codigo $=6626338$

Miranda, A. (2010). Educación secundaria, desigualdad y género en Argentina. Revista Mexicana de Investigación Educativa, 15(45), 571-598.

Ley $\mathrm{N}^{\circ}$ 5823. Ley Alumnos de Niveles Educativos Primario y Secundario Federados - Inasistencias - Condiciones (2017). Ciudad Autónoma de Buenos Aires, Argentina. 
Li, Ch. \& Wang, J.C.K. (2014). Talent development environmental factors in sport: a review and taxonomic classification. Quest, 66(4), 433-447.

Olovacha Toroshina, M. (2015). Diseño de una estrategia educativa para la selección de talentos deportivos en estudiantes de educación general básica (Tesis de maestría), Pontificia Universidad Católica del Ecuador, Quito. Recuperado de http://repositorio.pucesa.edu.ec/handle/123456789/1447

Rodríguez de La Cruz, J.C. (2016). Reconsiderando la exención en educación física para los deportistas de «alto nivel». Ágora para la Educación Física y el Deporte, 18(3), 230-243.

Siedentop, D. (1994). Sport Education. Champaign, IL: Human Kinetics.

Sport Northern Ireland. (2012). Sport policy factors leading to international sporting success: an audit of the elite sport development system in Northern Ireland. Belfast: Sport Northern Ireland. Recuperado de http://www.sportni.ne t/sportni/wp-content/uploads/2013/07/Splissreducedwebversion.pdf

Tuñón, I. y Di Paolo, G. (2018). Lo comportamental e institucional como factores asociados a las calificaciones escolares en Lengua y Matemática. Perflles educativos, 40(162), 86-99.

Tuñón, I. (2019). Infancias(s). Progresos y retrocesos en clave de desigualdad. Documento estadístico. Barómetro de la Deuda Social Argentina. Serie Agenda para la Equidad (2017-2025). Buenos Aires: Educa.

Velásquez, R. (2006). Dimensiones sociales y ambientales que influyen en la trayectoria de futbolistas juveniles. PubliCE Standard. Recuperado de https://g-se.com/es/psicologia-del-deporte/articulos/dimensiones-socialesy-ambientalesque-in-uyen-en-la-trayectoria-defutbolistas-juveniles-793

Vidosavljević, S. \& Krulj-Drasković, J. (2013). Effect between family and schools in detecting gifted children in sport. Activities in Physical Education and Sport, 3(2), 224-228. 